\title{
Temporomandibular disorders as an etiological factor for tinnitus
}

\author{
Andreeva R. ${ }^{1}$, Milkov M. ${ }^{2}$ \\ 'Department of Pediatric dentistry, Faculty of Dental Medicine, Medical University Varna \\ ${ }^{2}$ Department of Prosthetic Dentistry, Faculty of Dental Medicine, Medical University Varna
}

\begin{abstract}
Tinnitus is the sensation of hearing a sound with no external auditory stimulus present. Tinnitus is very prevalent and increases with increasing age. The most common presentation is high pitched cricket sounds, buzzing or ringing in both ears and often but not always associated with hearing loss. There are many possible causes of tinnitus, but few specific established treatments. Epidemiologic data indicate a frequent association between TMD and tinnitus. Disorders of the masticatory system may exert an influence on tinnitus via mechanical connections between the temporomandibular system and the ear or via neuronal influences. This hypothesis explains how the fusimotor system of the muscles innervated by the trigeminal motor nucleus is affected by inadequacies in the occlusion of the teeth that cause changes in posture and movement of the mandible. Inadequate occlusal contacts give rise to an adapted function of the mandible and the most common compensatory muscular response is hypertonia involving all mandibular muscles, including the tensor tympani. The treatment of tinnitus is very complex and etiologically defined. This article reviews the effect of TMD therapy on tinnitus.
\end{abstract}

\section{Introduction}

Tinnitus can affect the entire life of an individual, can prevent intellectual work, and impair the quality of life in general. Severe tinnitus is often accompanied by hyperacusis and affective disorders such as phonophobia and depression. [1] Tinnitus and auditory hallucinations are perceptions of sounds in the absence of external noise. [17] Tinnitus has many forms; it can be caused by sounds generated in the body (objective tinnitus) that reaches the ear through conduction in body tissue, but much more common is the tinnitus that occurs without any physical sound reaching the ear. Such tinnitus (subjective tinnitus) is a phantom sensation, where abnormal neural activity is generated in the ear, the auditory nerve, or the central nervous system. $[18,19]$ There are many forms of subjective tinnitus and it can occur with different severity. Subjective tinnitus often occurs in connection with hearing loss such as may occur after exposure to loud sounds (noise), or after administration of drugs such as certain antibiotics, but often no cause can be found. Some individuals who have severe tinnitus hear sounds as distorted and some have hyperacusis (reduced tolerance to sounds) [8] or phonophobia (fear of sounds) [7]. Tinnitus can be referred to one ear, or both ears, or to a location inside the head. The anatomical location of the physiological abnormality of chronic subjective tinnitus, however, is rarely in the ear but more often in the audi- 
tory nervous system. There are indications that the pathophysiology of unilateral and bilateral tinnitus is different.

\section{Aim}

The present study was designed to verify the correlation between tinnitus and temporomandibular joint dysfunction and treatment strategies of the TMD's in patients with tinnitus.

\section{Materials and methods}

35 consecutive patients were enrolled in the study, all affected by subjective tinnitus without hearing impairment, from both genders - 19 male and 16 female, age between 18 and 60 years old. All patients received a temporo-mandibular joint examination. All the patients were asked to rate the severity of their symptoms before and after treatment using a VAS scale and the Tinnitus Handicap Inventory (THI) and they followed a standardized protocol for the investigation of tinnitus. All the subjects were monitored by the same researcher as half underwent the same splint treatment and surgery and the other half underwent adjusting of deflective tooth contacts.

\section{Results}

The treatment methods included in the study are intraocclusal stabilization appliances, TMJ surgery and selective adjustment of the oclussal surface of teeth, followed by physiotherapy and medication. Most patients with TMD respond to conservative treatment and such reversible therapy does not change the structures of the masticatory system [26].The comparison between pre- and posttreatment phase scores showed in patients with predisposition of TMD and with TMD a statistically significant decrease of THI and VAS values. The characteristics of tinnitus and the degree of response to treatment confirmed the relationship between tinnitus and TMD. Occlusal splints and surgery showed remission or improvement in $86 \%$ of patients, with $31 \%$ complete remission and 55\% improvement respectively. Adjusting of occlusal contacts had $75 \%$ complete remission among patients.

\section{Discussion}

The authors believe that, when the most common causes of tinnitus, such as otologic disorders and neurological diseases are excluded, it is correct to evaluate the functionality of the temporo-mandibular joint and eventually treat its pathology to obtain tinnitus improvement or even resolution. [4] Otological symptoms in connection with TMD have been widely described in the literature $[11,12,20,22]$. Treatment of the TMD can successfully alleviate or cure tinnitus symptoms. [6,24]. The structures of the middle ear and the temporomandibular joint derive from the first branchial arch. All muscle of the masticatory system and the tensor tympani muscle and the tensor veli palatine muscle are innervated by branches of the trigeminal nerve. The ontogenetic development of the masticatory system and the middle ear associates a close anatomical relationship. There is a fibrous connection between the discal apparatus of the temporomandibular joint and the malleus of the middle ear $[10,15]$. By this connection pathologies of the TMJ may theoretically cause dysfunction of the middle ear, which in turn could cause tinnitus. Neuromuscular dysfunction of the masticatory muscles may induce a ,reflex hypertonia" of the tensor muscles of the middle ear [2]. The irregular tonus of the tensor veli palatini may result in a dysfunction of the Eustachian tube, which can result in aural congestion and tinnitus $[16,27]$. Removable occlusal applications made of resin have been recommended to relax masticatory muscles to eliminate or alleviate hearing symptoms triggered by TMJ disorders. Up to now occlusal appliances have not been proven to reduce or eliminate masticatory muscle dysfunction such as bruxism. Some autors believe that a reconstruction of the occlusal contacts of mandibular teeth, in association with maxillary teeth, may cure masticatory muscle spasms. [5,9,21]. Many individuals can modulate their tinnitus by movements of the head and neck. The contraction of facial mimic muscles [25], as well as also clenching the jaw or moving the head and neck, can cause change in tinnitus. The most common change is increase or decrease in loudness $[13,14]$. If tinnitus can be modulated by manipulation of somatosensory afferents from head and neck structures, dentists may contribute to the treatment of tinnitus. Dentists and maxillofacial surgeons have long known that tinnitus symptoms are not uncommon in patients with TMJ and masticatory muscle disorders (TMD). Clinicians who treated patients with TMD as a main complaint have noted that these patients often present with ear symptoms as secondary complaints. Therefore, related conditions such as tinnitus were improved 
and often eliminated after treatment of their TMJ problems. The incidence of TMD was found to be higher in patients with tinnitus than in the general population. TMD related tinnitus has been classified as objective tinnitus in most studies. From a dental perspective, tinnitus is possibly a secondary complaint of TMD or vice versa. Therefore, evaluation of possible involvement of the TMJ and masticatory muscle disorders seems feasible in all patients with tinnitus, as well as using TMD therapy in patients with TMD symptoms. It has been emphasized that the position of the mandible and the hyoid may influence the posture of the head and the cervical spine [23]. Occlusal changes during dental treatment may alter the resting position of the hyoid and the mandible. If the occlusion contacts in the resting position shift the mandible into a protruded, retruded or laterally displaced position, a muscular imbalance may occur, not only in the chewing muscles but in the muscles of the cervical spine as well. Thus imbalance of the occlusal system may result in abnormal somatosensory input to the DCN from the second cervical nerve and the trigeminal, which in turn modulates neuronal activity in other parts of the central auditory pathways. This mechanism is assumed to account for the association between head and neck pathologies and tinnitus. Many patients with tinnitus, who are referred to a dentist, have chronic tinnitus. The success of treatment of TMD-related tinnitus decreases with the duration of the tinnitus [3]. While therapy of acute TMD-related tinnitus may be aimed at total elimination, or significant reduction of the tinnitus the likelihood of complete remission of tinnitus is small in patients who have had their tinnitus for a long time [3]. Patients with objective tinnitus related to the TMJ or masticatory muscles (clicking and crepitation) are distinguished from those with subjective tinnitus. Treatment of objective tinnitus should focus on localizing and eliminating the source of the sound.

\section{Conclusion}

Complete remission of tinnitus remains an unrealistic therapeutic goal. At best, the dental approach can provide an additional therapeutic option in individual patients with tinnitus. TMD treatment options contribute to inter- and multidisciplinary approaches to the clinical management of the symptoms of tinnitus.

\section{Bibliography}

1. Георгиева Г, Симеонова Н, Влиянието на тинитуса върху качеството на живот, Втора научна конференция с международно участие „Здравето - предизвикателство пред професионалистите по здравни грижи“, 2019, с. 59.

2. Arlen H (2009) The otomandibular system. Clinical Management of Head, Neck and TMJ Pain Dysfunction. Philadelphia: WB Saunders and Co, 171-80.

3. Bosel C, Mazurec B, Haupt H, Peroz I (Chronic tinnitus and craniomandibular disorders Effectiveness of functional therapy on perceived tinnitus distress) HNO 2008, 56: 707-713.

4. Borissov B, Milkov M, Dimova M, Andreeva-Borisova R, Investigation of the relationship between tinntus and temporomandbular, disorders, Journal of the Union of Scientists - Varna, Medicine and Ecology Senes, 2 2019, 24: 31-35.

5. Dawson PE (1973) Temporomandibular joint pain-dysfunction problems can be solved. J Prosthet Dent 29: 100-12.

6. Gelb H, Calderone JP, Gross SM, Kantor ME (1967) The role of the dentist and the otorynolaryngologist in evaluating temporomandibular joint syndromes. J Prosthet Dent 18: 497-503.

7. Hazell, JWP, Sheldrake, JB, and Graham, RL. Decreased sound tolerance: predisposing factors, triggers and outcomes after TRT. In: Patuzzi R (ed) Proceedings of the Seventh International Tinnitus Seminar 2002. Perth, University of Western Australia, 2002: 255-261.

8. Jastreboff, PJ and Hazell, JWP. Tinnitus Retraining Therapy. Cambridge, Cambridge University Press, 2004.

9. Kerstein RB, Farell S (1990) Treatment of myofacial pain dysfunction syndrome with occlusal equilibration. J Prosthet Dent 63: 695-700.

10. Komori E, Sugisaki M, Tanabe H, Katoh S (1986) Discomalleolar ligament in the adult human. Cranio 4: 299-305.

11. Koskinen J, Paavolainen M, Raivio M, Roschier J (1980) Otological manifestations in temporomandibular joint dysfunction. J Oral Rehabil 7: $249-54$

12. Kuttila S, Kuttila M, Le BY, Alanen P, Suonpaa J (2005) Recurrent tinnitus and associated ear symptoms in adults. Int J Audiol 44: 164-70.

13. Levine RA (1999) Somatic (craniocervical) tinnitus and the dorsal cochlear nucleus hypothesis. Am J Otolaryngol 20: 351-62.

14. Lockwood AH, Salvi RJ, Burkard RF (2002) Tinnitus. N Engl J Med 347: 904-10.

15. Loughner BA, Larkin LH, Mahan PE (1989) Discomalleolar and anterior malleolar ligaments: possible causes of middle ear damage during temporomandibular joint surgery. Oral Surg Oral Med Oral Pathol 68: 14-22.

16. Malkin DP (1987) The role of TMJ dysfunction in the etiology of middle ear disease. Int J Orthod 25: 20-1.

17. Moller AR, Langguth B, DeRidder D, Kleinjung T, (2011) Textbook of Tinnitus, Chapter 1: 3-7. 
18. Moller AR, (2007) Tinnitus and pain, in Tinnitus: Pathophysiology and treatment, progress in brain research, B Langguth et al, Editors. 2007 Elsevier: Amserdam. 47-53.

19. Moller AR (1997) Similarities between chronic pain and tinnitus. Am. J. Otol. 18: 577-85.

20. Ogutchen-Toller M, Juniper RP (1993) The embryologic development of the human lateral pterygoid muscle and its relationships with the temporomandibular joint disc and Meckel's cartilage. J Oral Maxillofac 51: 772-8.

21. Ramfjord SP (1961) Bruxism, a clinical and electromyographic study. J Am Dent Assoc 62:21-44

22. Ramirez LM, Ballesteros LE, Sandoval GP (2008) Topical review: temporomandibular disorders in an integral otic symptom model. Int J Audiol 47: 215-27.

23. Rocabado M (1983) Biomechanical relationship of the cranial, cervical and hyoid regions. J Craniomandibular Pract 1: 61-6.

24. Vernon J, Griest S, Press L (1992) Attributes of tinnitus associated with the temporomandibular joint syndrome. Eur Arch Otorhinolaryngol 249: 93-4.

25. Yamamoto E, Nishimura H, Iwanaga M (1985) Tinnitus and/or hearing loss elicited by facial mimetic movement. Laryngoscope 95: 96670.

26. Zarb, GA, Carlsson, GE, Sessle, BJ, Mohl, ND, Temporomandibular joint and masticatory muscle disorders 1994, Munksgaard: Copenhagen

27. Zipfel TE, Kaza SR, Greene JS (2000) Middle-ear myoclonus, J Laryngol Otol 114: 207-9. 